Journal of Computer Science 8 (2): 200-204, 2012

ISSN 1549-3636

(C) 2012 Science Publications

\title{
Fuzzy Velocity Biased-Ad Hoc on Demand Distance Vector to Improve Throughput in Dynamic Mobile Network
}

\author{
${ }^{1}$ Tamizarasu, K. and ${ }^{2}$ M. Rajaram \\ ${ }^{1}$ Department of CSE, Jayam College of Engineering and Technology, \\ Dharmapuri, Tamil Nadu, India \\ ${ }^{2}$ Department of EEE, Anna University of Technology, \\ Tirunelveli. 627 007, Tamil Nadu, India
}

\begin{abstract}
Problem statement: With the escalating demand for mobile ad hoc network projects, major challenges to system planners and designers were addressing stability and reliability problems due to frequent change of network topology. Controlling an overhead, which means bandwidth utilization due to control packets, depends on the routing protocol, topology and data traffic. Limiting the network control overheads, of this highly dynamic and dense network to acceptable level, was closely linked to the function of increased link changes. The throughput of the network can be improved with dynamically limiting of the routing control overheads. Approach: We propose a improved AODV mechanism Fuzzy Velocity Biased-Adhoc on Demand Distance Vector (FVB-AODV) which dynamically modifies the AODV control overhead parameters based on the relative velocity between two communicating nodes. Conclusion/Recommendation: The proposed algorithm is able to achieve its objectives by increasing the throughput at high node mobility. Dynamically modifying the overheads improved the bandwidth utilization. The additional overheads on the node CPU however needs to be evaluated.
\end{abstract}

Key words: ad hoc network, MANET routing, ad hoc on demand distance vector, Temporally Ordered Routing Algorithm (TORA), fuzzy velocity, dynamic mobile network

\section{INTRODUCTION}

Mobile Ad hoc Network (MANET) is infrastructure less networks of mobile nodes linked with wireless connectivity (Corson and Macker, 1999), which are dynamic in nature, to transfer data. Wireless mobile networks based on cellular concept depends on infrastructure support of base stations which acts as access points to the mobile devices to route messages to and from mobile nodes in specified transmission area. WLAN, GSM, WLL etc are wireless networks based on this concept. Whereas MANET does not require any pre-existing fixed network infrastructure; centralized message passing device is not required for communicating between mobile nodes. MANET does not rely on any devices other than two or more mobile self-configuring, form their own network and transfer data packets to and from each other.

MANET finds wide applications in military, business and academia due to its infrastructure less, dynamic nature and the ease of deployment (Kalaavathi and Duraiswamy, 2007). A MANET either operates as nodes to cooperate to form network; mobile nodes are

a standalone or as a stub network. The mobile nodes act as host and router, each node acts as a router to forward packets between two or more nodes. The nodes are free to move and organize themselves in arbitrary manner, new nodes get included in the network, existing nodes may move out of the network thus the topology of the network keeps changing. Due to this dynamic nature of the MANET, it is a challenge to maintain the correct routes. The routing protocol used should be able to relay the traffic dynamically and be capable of adjusting to high/low mobility with high/low bandwidth.

Based on the way routes are created, the routing protocols can be divided into reactive protocols and proactive protocols (Johnson et al., 2007). In reactive protocols the route between nodes is found only on demand. When topology information is not available, the source node floods the packet and the path pursued to reach destination node is used. The various reactive routing protocols include Dynamic Source Routing (DSR) Ad hoc on demand Distance Vector (AODV) (Perkins et al., 2003; Yujun and Lincheng, 2010), Temporally Ordered Routing Algorithm (TORA), Signal Stability Routing (SSR). The proactive routing

Corresponding Author: Tamizarasu, K., Department of CSE, Jayam College of Engineering and Technology, Dharmapuri, Tamil Nadu, India 
protocol maintains a topology of each node to all other network nodes, the route creation is done by periodic emitting of hello packets irrespective of data traffic between source and destination. Thus when a data packet is to be forwarded, the path to the destination is already defined. Destination Sequenced Distance Vector (DSDV), Optimized Link State Routing (OLSR), Topology Dissemination Based on Reverse Path Forwarding (TBRPF) are some of the proactive routing protocols. Reactive protocols are more efficient than proactive protocols as reactive protocols minimize control overhead and power consumption, as routes are discovered only on demand. But proactive routing protocols provide better quality of service as routes to every destination is always updated thus reducing the end-to-end delay. It is studied and found that proactive routing protocols are best used in small scale static network and reactive routing protocol for medium sized network with moderate mobility (Belding-Royer and Toh, 1999).

The classifying the routing protocols based on the type of cast property, the protocols are divided as unicast, geocast, multicast and broadcast forwarding (Kuosmanen, 2010). Unicast forwarding is widely used in ad hoc networks, where one source transfers data packets to one destination. Multicast routing protocol when a node sends data packets to multiple destinations. Nodes are included and excluded as desired in multicast forwarding. Geocast forwarding is special type of multicast routing with a difference that the data packets are delivered to nodes present in specific geographical area. In broadcast forwarding the data packets are transmitted to all the neighbors located within one-hop from the source.

AD Hoc On demand Distance Vector routing (AODV) Protocol: AODV is based on the Bellmann Ford Distance Vector algorithm, adapting it to work in a mobile environment. It is a reactive routing protocol; a route to destination is discovered only on demand, when a node wants to forward a data packet to destination (Belding-Royer and Toh, 1999). The freshness of the route is maintained by use of sequence numbers to each of the routing information; it also ensures loop free routing.

Routing table: Each node in AODV contains a routing table, the routing table contains:

- Destination

- Next hop

- Number of hops to reach destination

- Destination sequence number

- Active neighbors for this route

- Route table entry expiration time

The expiration time depends on a parameter called active route timeout; it is reset every time the route has been used. The routing table is updated by using a series of control messages to determine and maintain routes.

Control messages: The different kinds of control messages used by this protocol are ROUTE REQUEST (RREQ), Route Reply (RREP) and Route Error (RERR) and Hello messages.

RREQ: Is a request message used when a source node wants to send data to a destination node and route is not available, it floods the network. The RREQ contains the following in the message:

Source Request Source Destination Destination Hop address ID Sequence No. address Sequence no. count

The request ID for each RREQ is unique, the node on receiving the RREQ checks the ID and if it had already received a RREQ in the same ID it will discard the request. Each node on receiving the RREQ updates the reverse route in the routing table to the source node. If the intermediate node does not carry valid route information in its routing table to the destination node, it re-broadcasts the RREQ. The number of RREQ messages that a node can send per second is limited.

RREP: Is a route reply message. When the RREQ arrives at the destination node, a reverse route is created or updated in routing table and a RREP is sent along the reverse route. The intermediate node on the reverse route updates the forward route to the destination node and passes along the RREP. The RREP contains the following in its message:

Source Destination Destination Hop Lifeaddress address sequence No count time.

The source code on receipt of RREP, on updating the path starts communication.

RRER: In AODV the route maintenance is done by each node monitoring their neighbor and RRER is generated whenever a node in an active route is not available. The RRER is sent to notify the neighboring nodes the loss of link and new route to destination is sought out.

HELLO messages: Nodes use HELLO messages to continuously monitor the local connectivity with other neighboring nodes that is the nodes it can directly communicate. The hello messages are never forwarded.

Sequence numbers: Helps keeping the route information fresh; outdated and unnecessary information are removed from the network. Sequence numbers prevent looping and also act as timestamp. 
The routing table stores the sequence number of all the destination nodes and updates whenever it receives the message with a greater sequence number.

ART: Active route timeout is the time until which the node removes the route state from the routing table. ART is the time at which the route is considered invalid.

The AODV routing protocol saves storage space and energy. AODV does not need a central administrative system to control routing process. Hello messages used for maintenance do not cause large overhead in the network. Topological changes in the network are fast as updates happen at only the affected nodes. AODV can be used even in low processing and low bandwidth utilization. The disadvantages are its latency and scalability.

Related works: Various developments have been proposed using AODV protocol; some of the researches done in this area are reviewed here.

An improved AODV for load balancing have been proposed (Rani and Dave, 2007) for route discovery using a new metric aggregate interface queue length. The conventional AODV was modified to provide a metric for load distribution, selection of light load path and reduce congested nodes. The proposed AODV shows an overall improvement in network life, throughput and end-to-end delay reduction.

AODV-BRL was proposed by Yujun and Lincheng (2010) to increase the higher packet delivery ratio and lower routing overhead by constructing a mesh structure to provide multiple alternate routes. Using AODV-BRL alternate routes are created using RREP packets and hello messages, extended routing table and the least hop count first to determine optimal alternative route.

Tomar (2008) proposed methods to improve performance in internet connectivity by applying local congestion methods in AODV. The packet delivery ratio increased even in constrained conditions when proposed method was applied. The results showed that the proposed method improved the normalized routing load by $27 \%$.

Kim et al. (2005) proposed a new routing algorithm to resolve the traffic of a node performance. The algorithm is called Selective Route Discovery (SRD) algorithm. In this algorithm, changes in network topology is quickly adapted as each nodes periodically send RREQ to frequently accessed nodes and the movement of the nodes noted. Experiments showed that for SRD algorithm has less packet delivery time and the traffic concentration for each destination node varies.

\section{MATERIALS AND METHODS}

The proposed protocol FVB-AODV is an extension of AODV and relies on the velocity of the nodes in the network. In a MANET the relative velocity between two nodes change continuously with the Received Signal Strength Indication (RSSI) changing dynamically. This is more obvious when the nodes are moving at high speed and away from each other. A sample relative movement trajectory model is illustrated in Fig. 1.

As the velocity of the node increases, the link reliability decreases. Let $=S_{a}$ and $S_{b}$ be the speed of two nodes $a$ and $b$. Let the link lifetime be denoted by $l_{\mathrm{ab}}$ between the nodes $\mathrm{a}$ and $\mathrm{b}$.

Let $R_{a b}$ denote the relative velocity between nodes $a$ and $b$.

The stability of the link can be determined using:

$$
1_{\mathrm{ab}}=\frac{\alpha}{\mathrm{R}_{\mathrm{ab}}}
$$

where, $\mathrm{a}$ is a constant.

The relative speed $S_{k}$ is given by:

$$
S_{k}=\frac{\left.\sqrt{\left(x_{k}-x_{k-1}\right.}\right)^{2}+\left(y_{k}-y_{k-1}\right)^{2}}{\text { Dist }}
$$

Two rules were introduced to reduce the control overheads in AODV

Rule 1: As the relative speed between two node increases, the route cache is flushed faster at a rate specified by the fuzzy inference engine from the base line of three seconds.

Rule 2: As the relative speed between two node increases, the intermediate node has to transmit an Route Reply (RREP) to the source node.

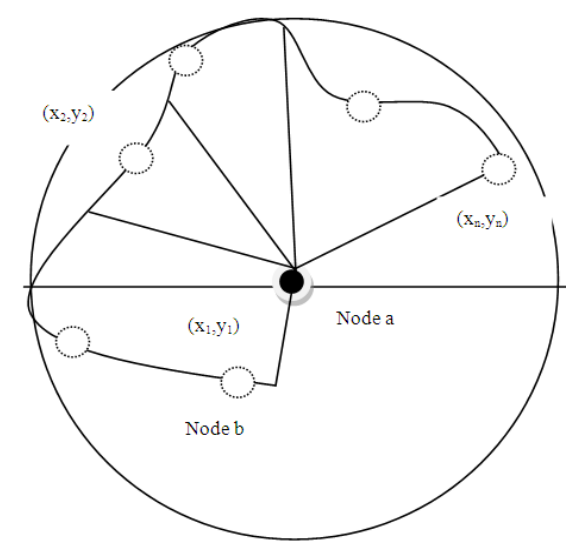

Fig. 1: Relative movement of node $b$ with respect to node a 


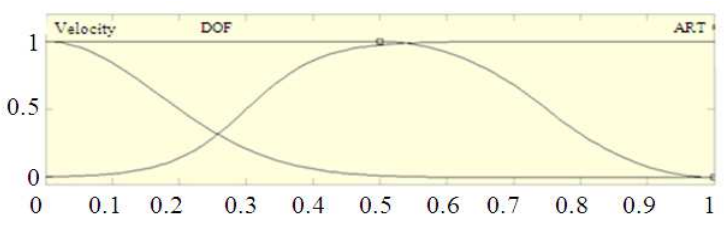

Fig. 2: The fuzzy membership function

Table 1: Simulation setup

Total area of the network

Number of nodes

Mobility of the nodes

$\begin{array}{ll} & \begin{array}{l}\text { speed of 100 Kmph in a } \\ \text { predefined circular route } \\ \text { 4 nodes moving at an average } \\ \text { speed of 65 Kmph in a } \\ \text { predefined straight route } \\ \text { Balance nodes moving } \\ \text { randomly about the network } \\ 1000 \mathrm{~m}\end{array} \\ \begin{array}{l}\text { Transmission range } \\ \text { of each node }\end{array} & 1 \mathrm{Mbps} \\ \begin{array}{l}\text { Data rate } \\ \text { Net diameter }\end{array} & 35 \\ \begin{array}{l}\text { Allowed hello loss } \\ \text { Network load }\end{array} & 3 \\ & \begin{array}{l}\text { Random generation and } \\ \text { transmission of data from each } \\ \text { node to all other nodes with } \\ \text { some nodes carrying voice } \\ \text { data to specific destination node }\end{array} \\ & \end{array}$

Since the status between a pair node changes over a period of time, we use a fuzzy inference system to change the parameters of the AODV as mentioned in rule 1 and 2 . The fuzzy mode used is the Sugeno fuzzy model with membership function values as shown in Fig. 2.

To verify the efficiency of the proposed mechanism, the proposed methodology was simulated using OPNET and compared with AODV. The simulation setup used is shown in Table 1.

\section{RESULTS}

Simulations were run for 20 minutes using AODV for base lining and the proposed protocol. The outputs recorded and shown from Fig. 3 through Fig. 4.

From Fig. 3 it is seen the routing overheads increasing in the network due to the increased RREP control packets sent due to low link stability. The overall bandwidth utilization increases, however the data transmission is more reliable.

Figure 4 shows the utilization of the caches globally across the network. As the utilization of the cache increases the route discovery time significantly comes down.

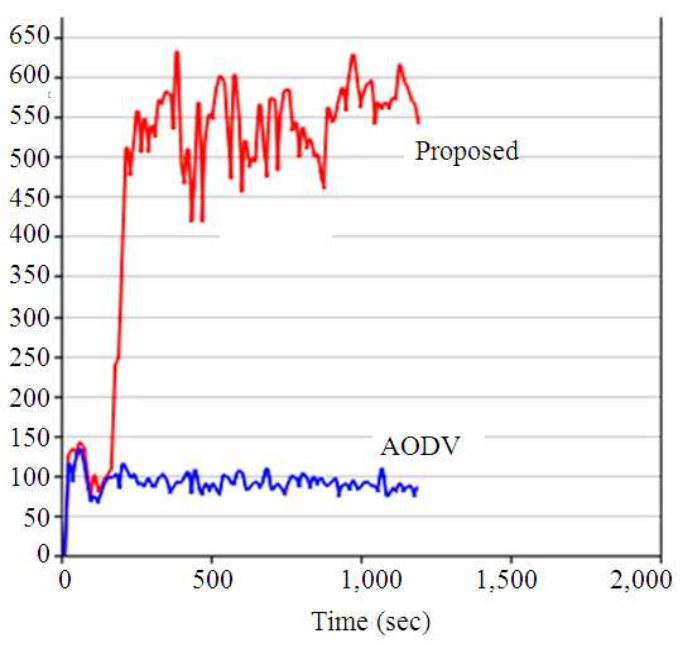

Fig. 3: Routing traffic received (packets/sec)

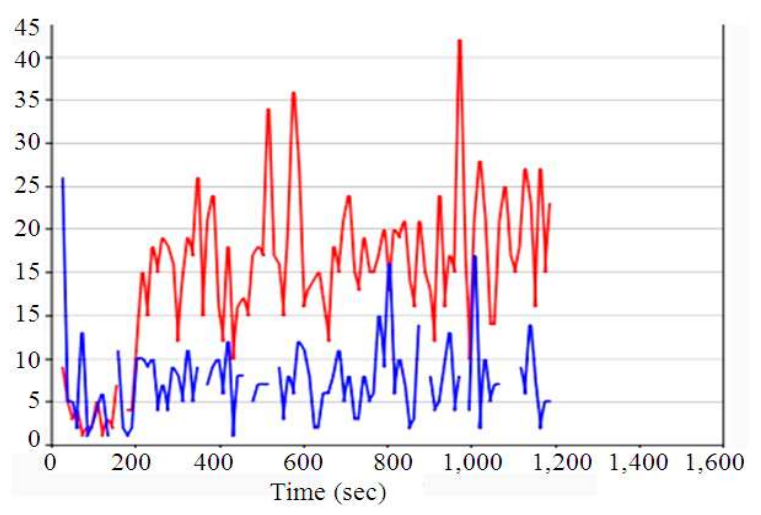

Fig. 4: Total route cache replies sent

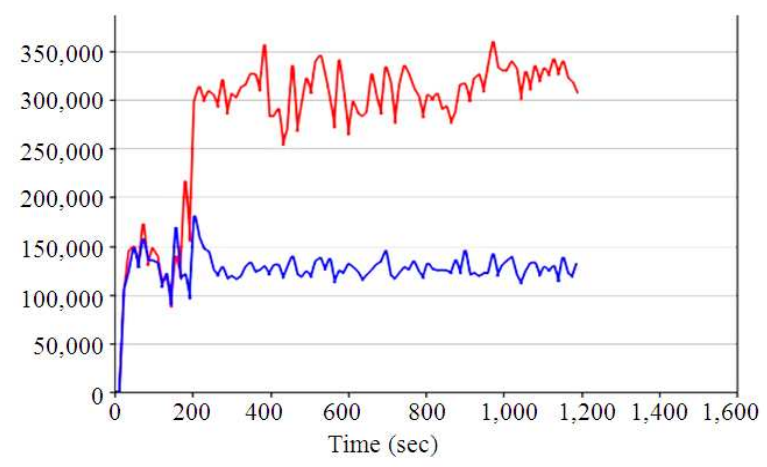

Fig. 5. Throughput in bits/second

Figure 5 shows the throughput of the network. It is seen that on an average the efficiency increases by a 95\% once the network becomes stable. 


\section{CONCLUSION}

In this study it was proposed to implement a novel dynamic routing algorithm based on AODV. The proposed algorithm uses fuzzy inference to dynamically vary the parameters of AODV and hence improve the throughput of the network. The proposed methodology was tested in a network containing nodes with different mobility speeds. The outputs obtained in network throughput is promising. Further work needs to be carried out for high volume data like video conferencing.

\section{REFERENCES}

Belding-Royer, E.M. and C.K. Toh, 1999. A review of current routing protocols for ad hoc mobile wireless networks. IEEE Person. Commun., 6: 4655. DOI: $10.1109 / 98.760423$

Corson, S. and J. Macker, 1999. Mobile Ad hoc Networking (MANET): Routing Protocol Performance Issues and Evaluation Considerations. The Internet Society.

Johnson, D., Y. Hu and D. Maltz, 2007. The Dynamic Source Routing protocol (DSR) for mobile ad hoc networks for IPv4. Rice University.

Kalaavathi, B. and K. Duraiswamy, 2008. Ant colony based node disjoint hybrid multi-path routing for mobile ad hoc network. J. Comput. Sci., 4: 80-86. DOI:10.3844/jcssp.2008.80.86
Kim, T.E., W.T. Kim and Y.J. Park, 2005. Selective route discovery routing algorithm for mobile adhoc networks. Inform. Network. Convergence Broadband Mobile Network., 3391: 152-159. DOI: 10.1007/978-3-540-30582-8_16

Kuosmanen, P., 2010. Classification of Ad Hoc Routing Protocols. The Pennsylvania State University.

Perkins, C., E. Belding-Royer and S. Das, 2003. Adhoc On-demand Distance Vector (AODV) routing. University of California.

Rani, A. and M. Dave, 2007. Performance evaluation of modified AODV for load balancing. J. Comput. Sci., 3: 863-868. DOI:10.3844/jcssp.2007.863.868

Tomar, G.S., 2008. Modified routing algorithm for AODV in constrained conditions. Proceedings of the 2nd Asia International Conference on Modelling and Simulation, May, 13-15, IEEE Xplore Press, Kuala Lumpur, pp: 219-224. DOI: 10.1109/AMS.2008.196

Yujun, L. and H. Lincheng, 2010. The research on an AODV-BRL to increase reliability and reduce routing overhead in MANET. Proceedings of the International Conference on Computer Application and System Modeling, Oct. 22-24, IEEE Xplore Press, Taiyuan, pp: 526-530. DOI: 10.1109/ICCASM.2010.5622386 